\title{
Dossografia dialettica e storia critica della filosofia in Aristotele. Tre esempi nella Metafisica ${ }^{1}$
}

\author{
Cristina Viano (Paris-Sorbonne/ CNRS)
}

Aristotle's "dialectical doxography", i.e. the analysis of opinions of ancient philosophers, is the first form of critical historiography in Antiquity. Through three examples from Metaphysics, we will attempt to demonstrate, against accusations of partiality directed at him, that Aristole was amicable to a historical perspective in the modern sense.

\section{La dossografia dialettica di Aristotele}

Benchè erede diretta della dialettica di Platone, la dialettica aristotelica appare come qualcosa di completamente nuovo sia per quanto riguarda il suo statuto di disciplina che il suo campo di applicazione.

Per Platone la dialettica era infatti una vera e propria scienza (epistêmê), anzi la più alta tra la scienze, la filosofia stessa. In Resp. VII, 533 ss., Platone dice appunto che la dialettica non è solo scienza, ma è il culmine, il coronamento (thrinkos) di tutte le scienze. Aristotele invece nega lo statuto di scienza alla dialettica e le attribuisce quello di un metodo (methodos), che permette di argomentare attorno a qualunque problema proposto.

Inoltre, per Platone la scienza dialettica ha come ambito il vero, l'essenza, le Forme intelliggibili. Per Aristotele, il campo della dialettica è l'opinione, il verosimile, il probabile, il per lo più; essa non costruisce ragionamenti scientifici fondati su principi veri ma ragionamenti dialettici, fondati su opinioni comuni largamente condivise. Non ha un oggetto specifico ma permette di trattare qualunque argomento, ha quindi un ruolo che potremmo dire trasversale.

\footnotetext{
${ }^{1}$ Questo testo si fonda su due conferenze, presentate rispettivamente all'Università di Venezia Ca' Foscari nell'ottobre 2008, durante le giornate di studio "La filosofia antica e i suoi metodi" e all'Università di Bergamo, nel dicembre 2010, al convegno "Filosofia prima e dialettica". Ringrazio gli amici e i colleghi presenti per i loro commenti e osservazioni.
} 
Journal of Ancient Philosophy Vol. V 2011 Issue 2

Ma Aristotele teorizza lo statuto e il valore epistemologico della dialettica in modo tale da mostrare che se non è scienza, essa è tuttavia estremamente utile alle scienze filosofiche. Essa infatti offre una via per accedere ai principi primi indimostrabili delle scienze attraverso l'esame delle "opinioni notevoli" (endoxa) esistenti su ciascuno di questi principi (dia de tôn peri hekasta endoxôn ... dielthein, Top. I, 2, 101 b1). Questa, dice Aristotele, è la funzione propria per eccellenza della dialettica: la funzione "esaminatrice" (exetastikê).

Ora, proprio della dialettica è la costruzione delle "dossografie", ovvero quelle esposizioni di opinioni (doxai) che si trovano all'inizio delle principali costruzioni teoriche del sistema aristotelico.

Vorrei ora soffermarmi proprio su questa applicazione particolare della dialettica aristotelica: la "dossografia dialettica", ovvero l'esame delle opinioni dei filosofi del passato che costituisce la prima forma articolata di storiografia filosofica dell'antichità. E vorrei mostrare che si tratta anche di storiografia critica, e che Aristotele, contrariamente alle accuse di parzialità che gli sono state rivolte, mostra di avere il senso della prospettiva storica in senso moderno e di prestare attenzione alla specificità delle dottrine e alla coerenza interna del discorso dei predecessori. A questo proposito, faro' tre esempi, tratti tutti dalla Metafisica: il celebre passo di A 3 sulla "scoperta" della causa materiale, le riflessioni di A 8 sulla scelta del fuoco come elemento per eccellenza e infine la valutazione dell'antica concezione "sotto forma di mito" delle divinità astrali di Lambda 8.

\section{Dialettica e endoxa}

Aristotele è il primo filosofo che fissa la sua concezione della dialettica in un trattato specifico, i Topici, a cui si aggiungono come appendice gli Elenchi Sofistici. Ora, nelle prime righe dei Topici, leggiamo:

Cio' che si propone questa trattazione è di trovare un metodo (methodos) per mezzo del quale saremo in grado di argomentare (sullogizesthai) a proposito di ogni problema proposto, a partire da opinioni notevoli (ex endoxôn), e dovendo noi stessi sostenere un discorso, non diciamo nulla di contrario a cio' che sosteniamo. (Top. I, 1, 100 a 18-21)

In questo passo troviamo riassunte le caratteristiche fondamentali della dialettica:

(1) Si tratta di un metodo (e non di una scienza) che insegna ad argomentare su qualunque problema. Ha quindi un carattere di universalità che la distingue dalle 
Journal of Ancient Philosophy Vol. V 2011 Issue 2

scienze particolari che hanno invece un oggetto determinato.

(2) La dialettica deve argomentare, cioè dedurre conseguenze (sullogizesthai).

Questo comporta due operazioni: da un lato criticare chi sostiene una certa tesi come soluzione del problema posto, dall'altro a difendersi da chi la critica quando la sosteniamo noi stessi. Colui che attacca deve argomentare da premesse concesse da chi sostiene la tesi, mentre colui che risponde deve fare attenzione a non concedere premesse da cui il suo avversario potrebbe dedurre una contraddizione e quindi cercare di non farsi confutare. Questo doppio movimento della dialettica ne mette in luce il carattere prettamente agonistico. Ma si tratta di un combattimento secondo regole ben precise, che riguardano la correttezza della deduzione e la natura delle premesse. Infatti la deduzione è corretta solo se la conclusione deriva necessariamente dalle premesse, quale sia il valore di verità di queste. Si puo' dire che la correttezza della deduzione garantisce se non la verità, almeno la coerenza di una posizione, nella compatibilità delle varie proposizioni che la costituiscono e nella loro concatenazione necessaria.

(3) Le premesse a partire da cui si deve argomentare devono essere endoxa, ovvero "opinioni notevoli". Ora, gli endoxa costituiscono una nozione importante, un termine tecnico proprio della dialettica aristotelica, su cui molto è stato scritto e sul cui impiego da parte di Aristotele nell'insieme del corpus regnano ancora interpretazioni constrastanti. Si puo' riassumere la discussione in due questioni principali ${ }^{2}$.

(a) La prima riguarda il significato di endoxon. All'inizio dei Topici, Aristotele indica come endoxa le premesse del sillogismo dialettico e le definisce secondo due criteri equivalenti e non esclusivi: uno quantitativo, fondato sull'estensione dell'accordo e l'altro qualitativo, fondato sull'autorità di chi lo sostiene: "le opinioni (dokounta, da dokein, sembrare, da cui deriva doxa, opinione) condivise da tutti o dai più o dagli esperti, e tra questi da tutti o dai più o dai più noti e famosi (endoxoi, lett. 'in fama')" (100b 21-23).

Il termine endoxos puo' essere interpretato in due modi, a seconda della connessione semantica che si stabilisce con $d o x a^{3}$. Se si intende doxa come "opinione", allora si traduce endoxos con "probabile, possibile, opinabile, plausibile", et quindi gli endoxa diventano "idee ammesse, fondate sull'opinione" ${ }^{4}$. Se invece si interpreta doxa

\footnotetext{
2 Per una panoramica molto utile dello status quaestionis, cfr. Seminara 2002. Di fatto, a mia conoscenza, non esiste ancora ad oggi una monografia completa e esaustiva sugli endoxa.

${ }^{3}$ Barnes 1980, p. 498 ss.

${ }^{4}$ Boezio (trad. lat. dei Topici, in Aristoteles lat. V, 1-3, ed. L. Minio-Paluello, Paris, 1969) fu il primo a tradurre endoxos con probabilis e opinabilis.
} 
Journal of Ancient Philosophy Vol. V 2011 Issue 2

nel suo secondo significato, e cioè di "fama", il termine significa "celebre, di buona reputazione, autorevole, notevole". In realtà, in greco è proprio questo il senso primo e più corrente di endoxos, che si ritrova anche nella definizione stessa data dai Topici. Come ipotizza giustamente J. Barnes, Aristote potrebbe essere stato il primo ad applicare l'aggettivo nel suo old sense a delle opinioni. Sulla base di questa interpretazione mi pare quindi che la migliore traduzione di endoxa sia "opinioni notevoli" (cfr. Zadro, Natali).

(b) La seconda questione riguarda la funzione che Aristotele attribuisce a queste opinioni notevoli nei differenti trattati e, in particolare, la legittimtà di considerare come endoxa non solo le premesse ma anche le tesi che sono oggetto di discussione. La messa in discussione degli endoxa è indicata da Aristotele stesso nel celebre passo metodologico di Eth. Nic. VII, 1, 1145 b 2-7, che precede la discussione sull'akrasia :

Bisogna, come negli altri casi (epi tôn allôn), dopo aver stabilito cio' che appare vero (ta phainomena) e avere, per prima cosa, sviluppato le aporie, comprovare (deiknunai) al meglio tutte le opinioni celebri (endoxa) su tali passioni, o, se non è possibile, la maggior parte e le più importanti: nel caso che siano state risolte le difficoltà e si lascino sussistere (kataleipetai) le opinioni autorevoli, si sarà data una dimostrazione sufficiente.

Non intendo qui soffermarmi a lungo sull'analisi di questo passo che è stato ampiamente discusso dai commentatori. Mi limiterò a evocare una recente messa a punto di Jaap Mansfeld ${ }^{5}$, in cui egli distingue tra endoxa ("opinions d'autorité"), che bisogna testare e phainomena (ciò che si crede), che vengono posti come punto di partenza, affrancandoci così dall'obbligo di considerare gli endoxa come dei dati da salvare in toto a qualunque prezzo: queste opinioni notevoli possono essere citate, riformulate, riviste, accettate $\mathrm{o}$, al limite, eliminate, se esse presentano delle difficoltà che possono essere risolte solo sopprimendole. Mansfeld riconduce così a proporzioni più modeste l'autorità degli endoxa ma ne sottolinea al tempo stesso il ruolo costruttivo all'interno del metodo aristotelico, non dell'etica ma anche delle altri parti della filosofia, in cui essi vengono integrati secondo diversi gradi di elaborazione. ${ }^{6}$

A mio parere, il problema di scegliere fra premesse e oggetto di esame, in fin dei conti non sussiste perchè si puo' immaginare che gli endoxa che servono a costituire delle premesse siano sottomessi a un valutazione.

\footnotetext{
${ }^{5}$ Mansfeld 2011.

${ }^{6}$ Cfr. anche Zingano 2007, che sottolinea questo atteggiamento positivo di preservare (kataleipein) cosa c'è di vero in ogni credenza.
} 
Journal of Ancient Philosophy Vol. V 2011 Issue 2

\section{Dialettica e storia della filosofia: le dossografie}

Le dottrine degli altri filosofi, e quindi anche dei predecessori, hanno quindi un ruolo fondamentale nella dialettica aristotelica in quanto rientrano sia nelle premesse, come opinioni notevoli e condivise (endoxa), sia nell'oggetto di discussione, come tesi alternative dei problemi da discutere riguardo le singole scienze. In Metaph. B, 1, 995 a 24-27, Aristotele mostra appunto come le opinioni contarie degli altri filosofi rientrano nel processo di sviluppo delle aporie:

E' necessario, in relazione alla scienza di cui siamo in cerca, che noi esaminiamo i problemi, dei quali bisogna innanzitutto cogliere le difficoltà (aporêsai). Si tratta di problemi intorno ai quali alcuni filosofi hanno fornito soluzioni contrastanti e, oltre a questi, di altri problemi che sono stati finora trascurati. Ora, per chi vuol risolvere bene un problema (euporêsai), è utile cogliere bene le difficoltà (diaporêsai kalôs) che esso comporta: la buona soluzione finale è infatti lo scioglimento (lusis) delle difficoltà precedentemente accertate.

E infatti, Aristotele fa cominciare la trattazione dei grandi temi della sua filosofia con l'esame delle opinioni, delle doxai, dei predecessori. Queste raccolte di opinioni, comunemente chiamate nel gergo filosofico "dossografie" (da doxa, "opinione, dottrina" e graphein, "scrivere") ${ }^{7}$.

Le principali dossografie sono le seguenti: Metaph. A, 3-7 (sui principi e le cause degli esseri); De anima I, 2-5 (sull'anima), Phys. I, 2-4 (sui principi della generazione), De caelo, I, 1, 279b 4 (sulla non generazione e l'icorruttibilità del cielo), De gen. et corr. I, 1 (sulla generazione e la corruzione); Pol. II, 1 (sulle forme di comunità politica) ${ }^{8}$.

In questi contesti, Aristotele instaura coi suoi predecessori un vero e proprio dialogo, una vera e propria discussione dialettica: egli espone e critica le loro dottrine per costruire la sua filosofia. Bisogna osservare pero' che l'apporto di queste ricerche sul passato è essenzialmente problematico e negativo. Le singole dottrine dei predecessori sono quasi sempre identificate ad aporie che bloccano lo sviluppo dellindagine e confutate. Esse servono quindi nella maggior parte dei casi a fare piazza pulita delle

\footnotetext{
${ }^{7}$ In senso proprio, i "dossografi" sono gli eruditi di età ellenistica, come Stobeo e Diogene Laerzio, che riunirono in raccolte sistematiche le dottrine dei filosofi antichi, le loro sentenze e informazioni storiche e biografiche. Essi costituiscono una delle fonti più importanti della nostra conoscenza dei Presocratici.

${ }^{8}$ Cfr. Mansion 1960, che analizza i diversi gradi di negatività delle dossografie aristoteliche. Ad es. in Metaph. B e De coel. le opinioni sono presentate come aporie che bloccano l'avanzare della ricerca.
} 
Journal of Ancient Philosophy Vol. V 2011 Issue 2

difficoltà e a preparare il terreno alla pars construens.

Per esempio, nel De anima, la dossografia critica e aporetica del primo libro serve a preparare il terreno alla costruzione della definizione di anima del secondo libro 9 .

Tra queste "dossografie" pero' quella del primo libro della Metafisica costituisce un caso eccezionale e merita una attenzione tutta particolare perchè mette in luce, più di ogni altra, non solo il valore teorico e filosofico ma anche il valore "storico" della dossografia dialettica di Aristotele.

\section{Metaph. A: il primo trattato di storia critica della filosofia}

Come ha osservato Enrico Berti in un articolo dedicato proprio alla storiografia dialettica aristotelica ${ }^{10}$, l'analisi dei predecessori del primo libro della Metafisica è da considerare la più completa e la più classica esposizione aristotelica della filosofia precedente, un modello che resterà alla base non solo di tutte le esposizioni dossografiche successive ma anche delle più recenti storie della filosofia.

Infatti non siamo più qui nell'ambito di una scienza particolare, ma in quello della filosofia "generale", della metafisica, della filosofia prima che ha come oggetto l'essere e i suoi principi. Aristotele non si serve delle dottrine per costruire i principi di una scienza particolare ma le valuta alla luce di un criterio forte già stabilito altrove, nella Fisica: la teoria delle quattro cause. Dal momento che la filosofia è conoscenza delle cause, si tratta di valutare i predecessori alla luce della nozione stessa di filosofia. S. Mansion parla di "confirmation historique nécessaire".

La conoscenza delle cause riassume infatti lo scopo di tutta la ricerca:

E' chiaro, dunque che occorre acquistare la scienza delle cause prime (tôn ex archês aitiôn...epistêmên) : infatti, diciamo di conoscere una cosa quando riteniamo di conoscerne la causa prima. Ora, le cause vengono intese in quattro significati diversi (...). Queste cause sono stata da noi studiate adeguatamente nella Fisica, tuttavia dobbiamo prendere in esame anche coloro che prima di noi hanno

\footnotetext{
9 "La ricerca sull'anima richiede che, insieme all'esame delle difficoltà che si devono risolvere (diaporountas peri ôn euporein) nel corso della trattazione, si raccolgano le opinioni (doxai) dei predecessori che si espressero in qualche modo intorno ad essa, e cio' per accogliere quanto hanno detto correttamente e evitare i loro eventuali errori. Diamo inizio all'indagine stabilendo le caratteristiche che, più di tutte sembrano appartenere all'anima per sua natura. Ora sembra che l'essere animato si distingua dall'inanimato specialmente per due proprietà: il movimento e la sensazione. Ed in verità anche dai nostri predecessori riguardo all'anima si puo' dire che abbiano appreso queste due sole caratteristiche" (I, 2, 403 b 20-29).
}

${ }^{10}$ Berti 1986, p. 112. 
Journal of Ancient Philosophy Vol. V 2011 Issue 2

affrontato lo studio degli esseri e hanno filosofato intorno alla realtà (peri tês alêtheias). E' chiaro, infatti, che anch'essi parlano di certi principi e di certe cause (archas tinas kai aitias). Ora, il rifarsi ad essi sarà certo un vantaggio alla presente trattazione: infatti o troveremo qualche altro genere di causa, oppure acquisteremo più salda credenza nelle cause di cui ora si è detto. (Metaph., A, 3, 983 a 24)

\section{(1) En hulês eidei}

La prima parte dell'analisi aristotelica delle dottrine dei predecessori sulle cause, è dedicata alla causa "materiale". Essa costituisce uno dei testi più fondamentali per la nostra conoscenza della filosofia presocratica.

Sul valore "storico", e quindi sull'attendibilità, delle informazioni date da Aristotele esiste da tempo un grande dibattito tra gli studiosi. Una delle accuse più gravi che sono state mosse a Aristotele è stata quella di aver deformato il pensiero dei suoi predecessori per costringerli nel letto di Procuste delle sue teorie, e in particolare in quella delle quattro cause ${ }^{11}$. Ora vorrei invece mostrare che Aristotele non si limita a appiattire le dottrine dei predecessori in uno schema prestabilito, ma che dà prova invece di possedere un certo senso della prospettiva storica e di attenzione alla coerenza interna delle doxai, nel presentare le versioni presocratiche della causa materiale. Vediamo come.

La maggior parte dei predecessori si sono sforzati di spiegare le cause del divenire ponendo come principi solo gli elementi corporei. Essi hanno riconosciuto unicamente la causa materiale e hanno identificato tutta la realtà al mondo materiale escludendo la causa formale.

La maggior parte di coloro che primi filosofarono pensarono che i principi (archai) di tutte le cose fossero solo quelli materiali (en hules eidei $=$ lett. in forma materiale). Infatti essi affermano che cio' di cui (ex hou) tutti gli esseri sono costituiti e cio' da cui (ex hou) derivano originariamente e in cui (eis ho) si risolvono da ultimo, è elemento ed è principio (stoicheion...archen) degli esseri, in quanto è una realtà che permane identica pur nel trasmutarsi delle sue affezioni. E, per questa ragione, essi credono che nulla si generi e che nulla si distrugga, dal momento che una tale realtà si conserva sempre. E come non diciamo che Socrate si genera in senso assoluto quando diviene bello o musico, nè diciamo che perisce quando perde questi modi di essere, per il fatto che il sostrato (to hupokeimenon) - ossia Socrate stesso - continua ad esistere, così dobbiamo dire che non si corrompe, in senso assoluto, nessuna delle altre cose:

\footnotetext{
${ }^{11}$ Cfr. Cherniss 1935, Intr. p. XII. Per lo status quaestionis, vedi Berti 1986.
} 
Journal of Ancient Philosophy Vol. V 2011 Issue 2

infatti deve esserci qualche realtà naturale (phusis) (o una sola o più di una) dalla quale derivano tutte le altre cose, mentre essa continua a esistere immutata.

Aristotele passa poi a descrivere le singole teorie dei predecessori "materialisti" su questo principio:

Tuttavia, questi filosofi non sono tutti d'accordo circa il numero e la specie (eidos) di un tale principio. Talete, iniziatore (archêgos) di questo tipo di filosofia, dice che quel principio è l'acqua (per questo afferma anche che la terra galleggia sull'acqua), desumendo indubbiamente questa sua convinzione (hupolêpsin labôn) dalla constatazione (horân) che il nutrimento di tutte le cose è umido, e che perfino il caldo si genera dall'umido e vive nell'umido. Ora, cio' da cui tutte le cose si generano è, appunto, il principio di tutto. Egli desunse dunque questa convinzione da questo fatto e dal fatto che i semi di tutte le cose hanno una natura umida e l'acqua è il principio della natura delle cose umide. Ci sono, poi, alcuni i quali credono che anche gli antichissimi (pampalaioi, i.e. Omero e Esiodo) che per primi hanno trattato degli Dei, molto prima della presente generazione abbiano avuto questa stessa concezione (houtôs hupolabein) della realtà naturale (peri tes phuseos). Infatti posero Oceano e Teti come autori della generazione delle cose, e dissero che cio' su cui gli dei giurano è l'acqua, la quale da essi vien chiamata Stige. Infatti, cio' che è più antico è anche cio' che è più degno di rispetto. Ma che questa concezione (doxa) della realtà naturale sia stata così originaria e così (984 a) antica, non risulta affatto in modo chiaro; al contrario, si afferma che Talete per primo abbia professato questa dottrina intorno alla causa prima (ché nessuno potrebbe pensare di mettere Ippone con costoro, a causa dell'inconsistenza del suo pensiero).

Anassimene, invece, e Diogene (di Apollonia) considerarono come originaria (proteron), più dell'acqua, l'aria e, fra i corpi semplici (hapla somata), la considerarono come principio per eccellenza, mentre Ippaso da Metaponto e Eraclito di Efeso, considerarono come principio il fuoco.

Invece Empedocle pose come principi i quattro corpi semplici, aggiungendo ai tre sopra menzionati anche un quarto, cioè la terra. Essi, infatti, restano sempre immutati e non sono soggetti a divenire se non per aumento o diminuzione di quantità, quando si congiungono in una unità o si sciolgono da essa.

Anassagora di Clazomene, che per étà viene prima di Empedocle ma è posteriore per le opere, afferma che i principi sono infiniti: infatti egli dice che pressoché tutte le omeomerie si generano e si corrompono unicamente in quanto si riuniscono e si disgiungono così come avviene per l'acqua o per il fuoco, mentre in altro modo non si generano nè si corrompono, ma permangono eterne. In base a questi ragionamenti, si potrebbe credere che ci sia una causa unica: quella che diciamo causa materiale (aitian ... en hules eidei). (983 b 6-984 a 18)

Vorrei sottolineare due punti in particolare che mi sembrano particolarmente significativi. (1) In primo luogo, si noterà che Aristotele non attribuisce ai predecessori la scoperta del suo concetto di causa materiale, ma che egli valuta i principi da loro ammessi (aria, acqua, terra e fuoco) come cause che dal suo punto di vista sono 
Journal of Ancient Philosophy Vol. V 2011 Issue 2

materiali. Questo principio (archê) è presentato come l'equivalente della causa materiale, ma è in realtà una entità sui generis ("certe cause") dotata di caratteristiche proprie : è autonoma, autosufficiente, ilomorfica, ilozoica, presenta un tipo di causalità più estesa benchè indeterminata rispetto alla causa puramente materiale di Aristotele: possiede una forza generatrice in sè, come l'acqua di Talete e come Oceano e Teti degli "antichissimi". Aristotele non la chiama certamente "materia", ma la indica con i termini "natura" (phusis), "elemento" (stoicheion), "principio" (archê).

Talete è l'archêgos di tale filosofia, Aristotele ricostruisce il percorso della scoperta di Talete: è il primo perchè è arrivato alla nozione di causa attraverso un ragionamento partendo dall'osservazione sensibile.

(2) Aristotele sembra poi distinguere due fasi nella storia della ricerca dei principi naturali. Vi sarebbe stata una fase pre-filosofica di antichissimi teologi, "molto prima della presente generazione" (polu pro tês nun genesê̂s). L'espressione "ci sono, poi, alcuni i quali credono che anche gli antichissimi..." fa anche pensare che Aristotele alluda a qualcuno che ne aveva già raccolto le opinioni. La fase filosofica, propriamente detta sarebbe stata poi inaugurata da Talete, come abbiamo visto.

Vorrei ora presentare gli altri due esempi, sempre nell'ambito delle cause e dei principi, che mi sembrano emblematici della sensibilità storico-critica di Aristotele.

\section{(2) Stoicheiôdestaton}

Più avanti, in 8,989 a 1 , nel contesto della critica dei filosofi naturalisti monisti, che hanno considerato in maniera troppo semplicistica come principio uno dei quattro elementi senza aver riflettuto sul modo in cui si generano reciprocamente, Aristotele dice:

Infatti, da un certo punto di vista (tê men gar), sembrerebbe essere elemento in grado maggiore (stoicheiôdestaton) rispetto a tutti gli altri, quel primo dal quale si generano, per un processo di riunione, tutti gli altri; ma un siffatto elemento dovrebbe essere quel corpo che è composto di parti più piccole e che è più sottile. Percio' tutti coloro che pongono come principio il fuoco, parlerebbero nel modo più conforme a questo modo di ragionare (malista homologoumenôs an tô logô toutô legoien). Ma anche tutti gli altri filosofi riconoscono (homologei) che l'elemento originario dei corpi deve essere di questo tipo. Infatti, nessuno di coloro i quali ammisero un solo elemento ritenne che questo fosse la terra, evidentemente per la grandezza delle sue parti. (Metaph., A, 8,988b 34)

$\mathrm{E}^{\prime}$ interessante notare che qui Aristotele, pur rifiutando la tesi generale dei filosofi monisti materialisti, che identificano il principio a un solo elemento, valuta le loro teorie 
Journal of Ancient Philosophy Vol. V 2011 Issue 2

"dall'interno", secondo un criterio di coerenza. Un solo principio materiale, posto all'origine di tutte le cose, deve possedere delle caratteristiche fisiche ottimali per assumere quel ruolo, e cioè essere costutito di parti piccole e sottili, che favoriscano l'aggregazione che dà origine agli altri elementi. Per questo la scelta del fuoco come elemento primo sembra una scelta ottimale, la più conforme a questo modo di vedere le cose.

\section{(3) En muthou schêmati}

L'altro esempio si trova in Metaph. Lambda, 8, 1074 b 1, nel contesto della dimostrazione dell'esistenza di una molteplicità di sostanze soprasensibili motrici delle sfere celesti. Aristotele parla di una tradizione tramandata da quegli "antichi" e dagli "antichissimi", che abbiamo già incontrato in Metaph. A:

Degli antichi e degli antichissimi (para tôn archaiôn kai pampalaiôn) è stata tramandata ai nostri posteri una tradizione, in forma di mito (en muthou schêmati), secondo la quale sono questi gli dèi, e il divino circonda la natura tutta. Le altre cose sono state, poi, miticamente (muthikôs) aggiunte, per infondere persuasione nel popolo e per far osservare le leggi e il bene comune. Dicono, infatti, che gli dèi hanno forma umana e che sono simili a certi animali, e, a queste, altre cose aggiungono della stessa natura o analoghe. Di queste, se prescindendo dal resto, si prende solo il punto fondamentale (monon to prôton) : cioè l'affermazione che le sostanze prime (prôtas ousias) sono dèi, bisogna riconoscere che essa è stata fatta per divina ispirazione (theiôs). E poichè, come è verosimile (kata to eikos), ogni arte fu trovata e poi nuovamente perduta, bisogna ritenere che queste opinioni degli antichi si sono conservate fino ad ora come reliquie (hoion leipsana). Fino a questo punto, dunque, solamente, ci sono note le opinioni dei padri e degli antenati (patrios doxa kai hê para tôn prôton). Metaph. $\Lambda, 8,1074 \mathrm{~b} 1-14$ )

Dice Aristotele che altri dettagli sono poi stati aggiunti in seguito muthikôs per persuadere il popolo ${ }^{12}$. Aristotele invita a considerare il nucleo di verità che questa

\footnotetext{
${ }^{12}$ Cfr. Metaph.a,3, 994b32: " L'attenzione alle lezioni dipende dalle abitudini. Infatti esigiamo che si parli nel modo in cui siamo abituati; e cio' che non ci viene detto in questo modo non ci sembra appropriato ma, per mancanza di abitudine, ci sembra più difficile da comprendere e più estraneo. Cio' che è abituale, infatti, è familiare. E quale sia la forza dell'abitudine, lo mostrano le leggi, a proposito delle quali cio' che è fiabesco e puerile, a causa dell'abitudine, ha più forza che la loro conoscenza". Per illustrare la forza dell'abitudine, Aristotele fa l'esempio delle leggi, in cui gli elementi fiabeschi e puerili hanno più forza persuasiva che la conoscenza delle leggi. Cio' significa: ubbidiamo alle leggi più a causa della persuasione che deriva dalle favole che ci hanno raccontato da bambini che per la conoscenza precisa dei testi.

Dal punto di vista negativo di Metaph. $\Lambda, 8,1074 \mathrm{~b} \mathrm{3}$, si puo' capire perchè il fiabesco è connesso al puerile(ta muthôdê kai paidariôdê). Il bersaglio implicito è probabilmente Platone. Basta pensare ai peamboli delle Leggi. Platone introduce dei miti persuasivi, o dissuasivi, che
} 
Journal of Ancient Philosophy Vol. V 2011 Issue 2

dottrina contiene, al di là delle aggiunte e variazioni mitiche successive : che le sostanze prime sono divine, come una reliquia di una scienza originaria di ispirazione divina. Benchè Aristotele sostenga che l'espressione mitica non è adatta alla filosofia, egli non ne critica la forma legittima di mito usata dagli antichi e dagli antichissimi, dai teologi che sono ancora al-di-qua della filosofia vera e propria, cominciata con Talete (penso che Aristotele intenda in questo senso il fatto che Talete è l'archêgos di toiautê philosophia: la corretta concezione della causa da un ragionamento che parte dall'osservazione sensibile $)^{13}$ - ne rigetta piuttosto le forme aggiunte e abusive e ne ritiene il nucleo originario.

Questo passo è particolarmente interessante perchè mostra in atto la doppia concezione aristotelica del mito e la sua valutazione "storica". Infatti, da un lato, per Aristotele il muthos è un termine tecnico: è una forma di esposizione poetica, più precisamente è il principio e l'anima della tragedia (Poet. 1450 a 32). Nella Poetica il mito, e cioè il racconto, la fabula, l'intreccio, è il discorso che presenta delle azioni connesse secondo necessità e verosimiglianza con lo scopo di suscitare emozioni au deuxième degré, e cioè il timore e la pietà, e il piacere che ne deriva. D'altro lato, per Aristotele, l'uso del mito è abusivo in filosofia: egli critica l'uso della spiegazione causale attraverso il mito per le incoerenze e gli errori a cui va incontro, per esempio nell'attribuire a cio' che ha una natura eterna e incorruttibile le caratteristiche del mondo sensibile $^{14}$. Spesso l'oggetto delle sue critiche è Platone, anche se non lo nomina esplicitamente. Per esempio, in De caelo, II, 1, 284 a 15 segg. associa il mito degli antichi (tôn palaiôn muthos) secondo cui il mondo avrebbe bisogno di Atlante per la sua conservazione all'attribuzione successiva (husteron) al cielo di una "necessità animata" (ananchên empsuchon). Non è difficile qui riconoscere un'allusione al Timeo (34b).

presentano talora lo statuto di "leggi", come certi miti escatologici destinati a generare la paura della punizione nell'al di là. Per esempio, in IX, $872 \mathrm{~d} 2 \mathrm{ss}$, la dottrina degli antichi preti, a cui rimanda anche la legge sul parricidio (chi ha ucciso il padre, in una vita futura, sarà ucciso dai suoi propri figli) è presentata come una "legge. Si veda a questo proposito, Laks 2005, p. 146ss. Alessandro (in Metaph. 167, 17) cita come esempio di miti imposti dai legislatori, la nascita degli uomini dalla terra e il mito di Cadmo,il figlio di un re fenicio che fece spuntare gli uomini dalla terra dopo avervi seminato i denti di un drago (cfr. Platone, Resp. III, 414b-415d). Secondo Alessandro, questi miti servono a sviluppare l'amore degli uomini per la terra e a spingerli a combattere come se essa fosse la loro madre.

${ }^{13}$ Cfr. Metaph. A, 3, 983b 20; 984a 2.

${ }^{14}$ Cfr. anche Metaph. B, 4, 1000 a 18, a proposito della decima aporia, se i principi degli esseri incorruttibili e degli esseri corruttibili sono gli stessi, dove oppone "le sottigliezze delle favole" (tôn muthikôn sophizomenôn) ai ragionamenti per dimostrazione (para de tôn di' apodeixeôs legontôn). 
Journal of Ancient Philosophy Vol. V 2011 Issue 2

Altrove, nelle opere biologiche, la spiegazione delle cause en muthô è criticata poichè sostituisce una spiegazione indimostrabile che dovrebbe invece venire dall'osservazione. ${ }^{15}$ Qui invece salva una spiegazione mitica delle cause corretta: essa contiene un nucleo di verità perchè riconosce le sostanze prime come divine.

\section{Conclusioni}

La dialettica aristotelica è dunque anche il metodo della storia della filosofia. Aristotele ha un approccio dialettico della filosofia del passato proprio in virtù del dialogo che egli instaura con i suoi predecessori, inserendo le loro dottrine nelle aporie preliminari a ogni indagine o valutandole, come nel primo libro della Metafisica, alla luce delle proprie dottrine. Aubenque vede proprio nella pratica - sofistica - della discussione e nella progressione verso la verità non solitaria, ma controllata e "pungolata" ( cfr. aiguillon, p. 11) dalle obiezioni dell'interlocutore, l'origine comune della dialettica platonica e aristotelica ${ }^{16}$.

Questi esempi tratti dalla Metafisica, che riguardano le cause, i principi e le realtà sovrasensibili, mostrano una visione piuttosto positiva e costruttiva. La visione globale aristotelica del progresso del pensiero umano è, in fin dei conti, piuttosto ottimistica, come è spesso nell'Etica la valutazione della natura umana. Tutti contribuiscono anche con poco, anche con i loro errori, alla ricerca della verità. L'inizio del libro alpha elatton della Metafisica illustra molto bene questa situazione:

La ricerca dellla verità sotto un certo aspetto è difficile mentre sotto un altro è facile. Una prova di cio' sta nel fatto che è impossibile per un uomo cogliere in modo adeguato la verità, e che è altrettanto impossibile non coglierla del tutto: infatti, se ciascuno puo' dire qualcosa intorno alla realtà, e se singolarmente preso, questo contenuto aggiunge poco o nulla alla conoscenza della verità, tuttavia dall'unione di tutti i singoli contributi deriva un risultato considerevole. Cosicchè se, relativamente alla verità, le cose sembrano che stiano così come nel proverbio "Chi potrebbe mancare una porta?", allora, per questo aspetto, sarà facile; invece, il fatto che si possa raggiungere la verità in generale e non nei particolari, mostra la difficoltà di essa. (...) E' giusto essere riconoscenti non soltanto a coloro di cui si condividerebbero le opinioni, ma anche a coloro che si sono espressi più superficialmente: anch'essi hanno portato un contributo poichè si sono esercitati

\footnotetext{
${ }^{15}$ Cfr. Hist. anim 31, 579 b 4, sul mito dell'espulsione dell'utero delle leonesse durante il parto per spiegare la rarità dei leoni e 35,580 a 15 sul mito che le lupe partoriscono tutte insieme ogni anno, durante un periodo di dodici giorni.
}

${ }^{16}$ Cfr. Aubenque 1970. 
Journal of Ancient Philosophy Vol. V 2011 Issue 2

nella disciplina prima di noi (tên, gar hexin proêskêsan hêmôn). (Metaph.a 1, 992a 29 ss.)

La conclusione di Metaph. A sembra applicare questa visione alla ricerca delle cause:

Dunque, da cio' che sopra si è detto, risulta evidente che tutti i filosofi sembrano aver ricercato le cause da noi stabilite nella Fisica, e che non si puo' parlare di alcun'altra causa all'infuori di queste. Ma essi hanno parlato di queste cause in maniera confusa. $\mathrm{E}$, in un certo senso, tutte da loro sono state menzionate, mentre in un altro senso non sono state affatto menzionate. La filosofia primitiva, infatti sembra che balbetti su tutte le cose, essendo essa giovane e ai suoi primi passi.

(Metaph.A, 10, 993 a 11)

\section{Bibliografia}

Aubenque, P. "La dialectique chez Aristote", dans L'attualità della problematica aristotelica, Padova, Antenore, 1970, p. 9-31.

Barnes, J. "Aristotle and the Methods of Ethics", Revue internationale de Philosophie 34 (1980), p. 490-511.

Berti, E. "Sul carattere 'dialettico' della storiografia filosofica di Aristotele", in G. Cambiano (ed.), Storiografia e dossografia nella filosofia antica, Torino, Tirrenia Stampatori, 1986, p. 101-125.

Cherniss, H. Aristotle' Criticism of Presocratic Philosophy, Baltimora, 1935.

Mansfeld, J. "Nichomachean Ethics 1145 b 2-6", in C. Natali (ed.), Aristotle: Metaphysics and Practical Philosophy, Studies in Honour of Enrico Berti, Peeters Publishers, Louvain, 2011, p. 165-176.

Mansion, S. "Le rôle de l'exposé de la critique des philosophies antérieures chez Aristote" dans S. Mansion (éd.), Aristote et les problèmes de méthode, Louvain 1980 $\left(1961^{1}\right)$, p. 35-56.

Zingano, M. "Aristotle and the problems of method in Ethics", Oxford Studies in Ancient Philosophy, 32 ( 2007), p. 297-329. 